Working Paper 91-25

October 1990
Departamento de Economia Universidad Carlos III de Madrid Calle Madrid, 126

28903 Getafe (Madrid)

ON EIGENVALUES, CASE DELETION AND EXTREMES IN REGRESSION

Santiago Velilla*

Abstract

This paper presents an approximation for assessing the effect of deleting an observation in the eigenvalues of the correlation matrix of a multiple linear regression model. Applications in connection with the detection of collinearityinfluential observations are explored.

Key words: Case deletion; Collinearity; Eigenvalues; Extreme cases; Gateaux differentiability; Multiple Linear Regression; Perturbation theory.

* Departamento de Estadistica y Econometría. Universidad Carlos III de Madrid. 


\section{ON EIGENVALUES, CASE DELETION AND EXTREMES IN REGRESSION}

\section{Santiago Velilla}

Facultad de Económicas, Dpta. de Estadistica, Universidad Carlas III de Madrid, 28903-Getafe, Ypain.

This paper presents an approximation for assessing the effect of deleting an observation in the eigenvalues of the correlation matrix of a multiple linear regression model. Applications in connection with the detection of collinearity-influential observations are explored.

\section{Introduction}

Consider the linear model

$$
\mathrm{Y}=\mathrm{X} \beta+\varepsilon,
$$

where $\mathrm{Y}$ is an $\mathrm{n}$ vector of observable responses, $\mathrm{X}$ is a known full rank nxm matrix, $\beta$ is an $m$ vector of unknown parameters, and $\varepsilon$ is an $n$ vector of unobservable errors with $E[\varepsilon]=0$ and $V[\varepsilon]=\sigma^{2} I_{n}$. We assume that the model contains an intercept term and, therefore, $X=\left(1_{n}, X_{1}\right)$, where $1_{n}$ is an $n$ vector of ones and $X_{1}$ is an $n \times p$ matrix. We have $m=p+1$. Sometimes, it is convenient to write the model (1.1) as

$$
Y=1_{n} \alpha+Z \alpha+\varepsilon
$$

AMY 1980 subject classifications. Primary 62J05.

Keywards and phrases: Case deletion; Collinearity; Eigenvalues; Extreme cases; Gâteaux differentiability; Multiple Linear Regression; Perturbation theory. 
where $Z$ is the nxp matrix of centered and scaled predictors and $\left(\alpha_{0}, \alpha^{\prime}\right)^{\prime}$ is an $\mathrm{m}$ vector of unknown parameters.

According to (1.2), $\mathrm{Z}^{\prime} \mathrm{Z}$ is the correlation matrix of the model

(1.1). We write its spectral decomposition as

$$
Z^{\prime} Z=C D C^{\prime}
$$

where $C$, with columns $\gamma_{j}$, is a pxp orthogonal matrix of eigenvectors, and $D$ is a pxp matrix of eigenvalues $0<\lambda_{1} \leq \ldots \leq \lambda_{p}$. Closely related to (1.3) is the $\mathrm{nxp}$ matrix of principal components associated to (1.1), namely,

$$
K=\left(k_{1 j}\right)=Z C,
$$

with columns $\mathrm{k}_{\mathrm{j}}=\mathrm{Zr}_{\mathrm{j}}$.

A linear least squares fit of (1.1) may be affected by collinearity among the columns of $\mathrm{X}$. Collinearity refers to the near dependence of the regressor variates. Consequences of collinearity with regard to numerical and statistical instability of coefficient estimates are well documented (see Gunst (1983) for a detailed review). A useful indicator of the existence of a near linear dependence among the columns of $\mathrm{X}$ is the presence of small eigenvalues in D. Given that

$$
\lambda_{j}=\gamma_{j}^{\prime} Z^{\prime} Z \gamma_{j}=k_{j}^{\prime} k_{j}
$$

$\lambda_{\mathrm{J}} \cong 0$ implies $\mathrm{Zr}_{\mathrm{J}} \cong 0$. We define the jth condition index of the matrix $\mathrm{Z}$, $\eta_{j}=\left(\lambda_{p} / \lambda_{j}\right)^{1 / 2}$. In particular, $\kappa=\eta_{1}=\left(\lambda_{p} / \lambda_{1}\right)^{1 / 2}$ is the condition number of $z$. The set $\left\{\eta_{j}, 1 \leq \mathrm{j} \leq \mathrm{p}\right\}$ of condition indexes is a diagnostic tool for detecting collinearity proposed by Belsley et al. (1980).

The nxn matrix $\mathrm{V}=\left(\mathrm{v}_{1 \mathrm{j}}\right)=\mathrm{X}\left(\mathrm{X}^{\prime} \mathrm{X}\right)^{-1} \mathrm{X}^{\prime}$ is termed the hat matrix and its diagonal entries $v_{11}$ measure how far the associated cases are from the center of the data set. It is shown that $V=l_{n} l_{n}^{\prime} / n+Z\left(Z^{\prime} Z\right)^{-1} Z^{\prime}$ and then, using (1.3) and (1.4), 


$$
v_{11}=\frac{1}{n}+\sum_{j=1}^{p} \frac{k_{i j}^{2}}{\lambda_{j}} .
$$

Cases with $v_{n}>2 m / n$ are extremes or far from the bulk of the data.

It is well-known that a small group of cases might be distorting, either creating or masking, the perception of an approximate linear relationship among the columns of model (1.1). These points are dubbed collinearity-influential cases. For example, Mason and Gunst (1985) showed how a group of $k$ outlying cases can induce $k-1$ near linear relationships among the regressor variates. Collinearity-influential cases were initially noted in Belsley et al. (1980) and Cook and Weisberg (1982).

Several authors have proposed different techniques for detecting collinearity-influential points, among others, Chaterjee and Hadi (1988), Walker (1989) and, recently, Wang and Nyquist (1991). For a recent review on diagnostic techniques for collinearity-influential points, see Belsley (1991, chap. 8).

Put $x_{1}=\left(x_{1}, \ldots, x_{n}\right)^{\prime}$, where $x_{1} \in \mathbb{R}^{p}$. In what follows, the subscript (1) will mean that the corresponding quantity has been computed deleting the ith case $\left(1, x_{1}^{\prime}\right)$ of the analysis. Since collinearity measures are mainly constructed on the eigenvalues of the (centered ans scaled) cross products matrix, a method for diagnosing the presence of collinearity-influential points in a data set could be analyzing the relationship among the sets $\left\{\lambda_{j}\right\}$ and $\left\{\lambda_{j(1)}\right\}$. Unfortunately, with the exception of some special cases, there are no explicit expressions relating $\lambda_{J}$ to $\lambda_{J(1)}$ and, therefore, some degree of approximation seems in order. The aim of this paper is to present a new technique of approximation for the differences $\lambda_{J}-\lambda_{J(i)}$. The accuracy of the approximation is reflected in a rate of convergence which depends on the 
sample size. Section 2 contains some preliminary background to the main results which are exposed in section 3 . Section 4 is devoted to applications and examples. We will focus primarily on the effect of single deletion. Results concerning multiple cases and other possible extensions of the theory can be found in section 5 .

\section{Eigenvalues as functionals}

Let $\varphi_{\mathrm{m}}$ be the space of all real mxm symmetric matrices. For a given element $A=\left(a_{1 j}\right)$ in $\varphi_{m}$, we will take the norm $\|A\|=\|A\|_{\infty}=\max _{i, j}\left|a_{1 j}\right|$. We write the spectral decomposition of $\mathrm{A}$,

$$
A=C(A) D(A) C(A)^{\prime},
$$

where $C(A)$, with columns $g_{j}(A)$, is a mxm orthogonal matrix of eigenvectors, and $D(A)$ is a mxm matrix of eigenvalues $\ell_{1}(A) \leq \ldots \leq \ell_{m}(A)$. Let $F_{n}$ the distribution function in $\mathbb{R}^{p}$ of the design measure associated with the $n$ rows $x_{1}, \ldots, x_{n}$ of the matrix $x_{1}$. We have the integral representation

$$
X^{\prime} X / n=\int y y^{\prime} F_{n}(d y)=T\left[F_{n}\right],
$$

$y=\left(1, x^{\prime}\right)^{\prime} \in \mathbb{R}^{m}$, where $T[$.$] is an adequate functional defined in a space of$ measures. We can write $\ell_{j}\left(X^{\prime} X\right)=n \ell_{j}\left(\int y y^{\prime} F_{n}(d y)\right)=n \ell_{j}\left(T\left[F_{n}\right]\right) \quad$ and, consequently,

$$
\begin{gathered}
\ell_{j}\left(X^{\prime} X\right)-\ell_{j}\left(X_{(1)}^{\prime} X_{(1)}\right)= \\
\ell_{j}\left(T\left[F_{n}\right]\right)+(n-1)\left\{\ell_{j}\left(T\left[F_{n}\right]\right)-\ell_{j}\left(T\left[F_{n-1(1)}\right]\right)\right\},
\end{gathered}
$$

where $F_{n-1(1)}$ is the distribution function of the design measure on the $\mathrm{n}-1$ rows of $\mathrm{x}_{1(1)}$, namely,

$$
F_{n-1(1)}=F_{n}+\frac{1}{n-1}\left(F_{n}-\delta_{x_{1}}\right)
$$

We can take advantage of (2.3) and (2.4) to develop an approximation for the difference $\ell_{j}\left(X^{\prime} X\right)-\ell_{j}\left(X_{(1)}^{\prime} X_{(1)}\right)$ based on certain 
differentiabiliy properties of the functional $\ell_{j}(T[]$.$) . We make the$ following assumptions:

Assumption 1. The points $x_{1}, \ldots, x_{n}$ are the first $n$ elements of an infinite sequence $\left\{x_{1}\right\}$ of points contained in a compact set $C \subseteq \mathbb{R}^{p}$.

Assumption 2. Let $\mathcal{M}$ be the linear space of finite signed measures concentrated on C. The functional T[.] of (2.2) is defined in $M$ in the form $T[\mu]=\int y y^{\prime} \mu(d y) . \quad M$ is endowed with the norm $\|\cdot\|_{V}$ of the total variation (see Rudin (1974, chap. 6) for definition).

Observe:

a) Under assumption 1 , both $F_{n}$ and $F_{n-1(1)}$ are in $M$ and $\left\|F_{n}\right\|_{V}=\left\|F_{n-1(1)}\right\|_{v}=1$

b) The functional $\mathrm{T}:\left(M,\|\cdot\|_{\mathrm{V}}\right) \longrightarrow\left(\varphi_{\mathrm{m}},\|\cdot\|_{\infty}\right)$ is linear $(T[\alpha \mu+\beta \lambda]=\alpha T[\mu]+\beta T[\lambda], \quad \mu, \lambda \in \mathcal{M}, \quad \alpha, \beta \in \mathbb{R}) ; \quad$ and bounded $\left(\|T[\mu]\|_{\infty} \leq M\|\mu\|_{V}\right.$ for some fixed constant $\mathrm{M}>0$ independent of $\mu$ );

c) For $\mu \in M, \ell_{j}(T[\mu])$ means, in the ordering established above, the jth eigenvalue of $T[\mu]=\int_{C} y y^{\prime} \mu(d y)=\left(t_{i k}[\mu]\right) \in \varphi_{m}$. If $\ell_{j}(T[\mu])$ is simple, $h \in \mathcal{M}$ and $t \longrightarrow 0$, we get, from standard results of perturbation theory for real symmetric matrices (see Kato (1982) for details),

$$
\ell_{j}(T[\mu+\mathrm{th}])=\ell_{j}(T[\mu]+\mathrm{tT}[\mathrm{h}])=\ell_{j}(T[\mu])+\mathrm{t} q_{j}^{\prime}(T[\mu]) T[h] q_{j}(T[\mu])+o(|\mathrm{t}|) .
$$

This entails,

$$
\lim _{t \rightarrow 0} \frac{\ell_{j}(T[\mu+t h])-\ell_{j}(T[\mu])}{t}=D \ell_{j}(T[\mu])(h),
$$

where $D \ell_{j}(T[\mu])(h)=g_{j}^{\prime}(T[\mu]) T[h] g_{j}(T[\mu])$. Since $D \ell_{j}(T[\mu])(h)$ is linear in $h$, (2.5) says that $\ell_{j}(T[\mu]$ ) is Gateaux (weakly) differentiable at every $\mu \in \mathcal{M}$ such that the $j$ th eigenvalue of $T[\mu]$ is simple.

We now proof the following theorem. 
Theorem 1. Let $\mu, \lambda \in \mathcal{M}$ such that $\|\mu\|_{V^{\prime}}\|\lambda\|_{V} \leq 1$. If $T[$.$] has all its$ eigenvalues simple at every point of the segment $(1-t) \mu+t \lambda, 0 \leq t \leq 1$, then,

$$
\max _{1 \leq j \leq m}\left|\ell_{j}(T[\lambda])-\ell_{j}(T[\mu])-D \ell_{j}(T[\mu])(\lambda-\mu)\right| \leq Q(\lambda, \mu)\|\lambda-\mu\|_{V^{\prime}}
$$

where $\mathrm{Q}(\lambda, \mu) \longrightarrow 0$, if $\|\lambda-\mu\|_{\mathrm{v}} \longrightarrow 0$.

Proof. For each $j=1, \ldots, m$, the functional $\ell_{j}(T[]$.$) is weakly$ differentiable in all the segment $[\mu, \lambda]$. We can then apply the mean value theorem (see Lang (1969, chap. 5)) to obtain

$$
\max _{1 \leq j \leq m}\left|\ell_{j}(T[\lambda])-\ell_{j}(T[\mu])-D \ell_{j}(T[\mu])(\lambda-\mu)\right| \leq Q(\lambda, \mu)\|\lambda-\mu\|_{V^{\prime}}
$$

where $Q(\lambda, \mu)=\max _{1 \leq \mathrm{J} \leq \mathrm{m}} \sup _{0 \leq \mathrm{t} \leq 1\|\mathrm{~h}\|_{\mathrm{v}} \leq 1}\left|\left\{\mathrm{D} \ell_{\mathrm{J}}(\mathrm{T}[(1-\mathrm{t}) \mu+\mathrm{t} \lambda])-\mathrm{D} \ell_{\mathrm{J}}(\mathrm{T}[\mu])\right\}(\mathrm{h})\right|$.

To proof that $Q(\lambda, \mu) \longrightarrow 0$, as $\|\lambda-\mu\|_{V} \longrightarrow 0$, we reason as follows. As remarked in Kato (1982, p. 136), the eigenvalues $\ell_{J}(A)$ and eigenvectors $g_{J}(A)$ are uniformly continuous functions of matrix argument in any bounded region of $\varphi_{\mathrm{m}}$ (i.e. in any region of $\varphi_{\mathrm{m}}$ where $\|\mathrm{A}\|_{\infty}$ is bounded). Since $\|T[\lambda]-T[\mu]\|_{\infty} \leq M\|\lambda-\mu\|_{v^{\prime}}$ the eigenvalues $\ell_{j}(T[\nu])$ and eigenvectors $g_{J}(T[\nu])$ are uniformly continuous functions of $v$ in the region $\|v\|_{v} \leq 1$. On the other hand, we have, for $\|\mathrm{h}\|_{\mathrm{V}} \leq 1$, the elementary inequality $\left|a^{\prime} T[h] b\right| \leq m M\|a\|_{E}\|b\|_{E^{\prime}}$ where $\|\cdot\|_{E}$ denotes the euclidean norm in $\mathbb{R}^{m}$. It is easy to see that putting all these things together, we get the claim of the theorem.

As an example of possible applications of theorem 1, we establish the following corollary.

Corollary 1. Suppose that, for every $n$ and $1 \leq i \leq n$, the matrix $T\left[(1-t) F_{n}+t F_{n-1(1)}\right]$ has all its eigenvalues simple for $0 \leq t \leq 1$. Then,

$$
\max _{1 \leq j \leq m} \max _{1 \leq 1 \leq n}\left|\ell_{j}\left(X^{\prime} X\right)-\ell_{j}\left(X_{(i)}^{\prime} X_{(1)}\right)-k_{1 j}^{2}\left(X^{\prime} X\right)\right| \leq a_{n^{\prime}}
$$

where $k_{1 j}\left(X^{\prime} X\right)=\left(1, x_{i}^{\prime}\right) g_{j}\left(X^{\prime} X\right)$ and $a_{n} \longrightarrow 0$ as $n \longrightarrow \infty$. 
Proof. Put $\lambda=F_{n-1(1)}$ and $\mu=F_{n} \cdot B y$ (2.4) and (2.5) above, $D \ell_{j}\left(T\left[F_{n}\right]\right)(\lambda-\mu)$ equals,

$$
\left[\left(\ell_{j}\left(X^{\prime} X\right) / n\right)-k_{1 j}^{2}\left(X^{\prime} X\right)\right] / n-1
$$

Using (2.3), we get $\ell_{j}\left(X^{\prime} X\right)-\ell_{j}\left(X_{(1)}^{\prime} X_{(1)}\right)-k_{1 j}^{2}\left(X^{\prime} X\right)=$

$$
-(n-1)\left\{\ell_{j}\left(T[\lambda]-\ell_{j}(T[\mu])-D \ell_{j}\left(T\left[F_{n}\right]\right)(\lambda-\mu)\right\}\right. \text {. }
$$

We have $\|\lambda-\mu\|_{v}=\left\|\frac{1}{n-1}\left(F_{n}-\delta_{x_{1}}\right)\right\|_{v}=2 / n$. From theorem 1 above, $\max _{1 \leq j \leq m} \max _{1 \leq 1 \leq n}$ $\left|\ell_{j}\left(X^{\prime} X\right)-\ell_{j}\left(X_{(1)}^{\prime} X_{(1)}\right)-k_{1 j}^{2}\left(X^{\prime} X\right)\right| \leq(2 / n)(n-1) b_{n^{\prime}}$ where the sequence $b_{n}=\max _{1 \leq 1 \leq n} Q\left(F_{n-1(1)}, F_{n}\right)$ tends to zero as $n$ goes to $\infty$. The corollary follows with $a_{n}=(2 / n)(n-1) b_{n}$.

Remark 1. For practical purposes, (2.7) yields the approximation

$$
\ell_{j}\left(X^{\prime} X\right)-\ell_{j}\left(X_{(1)}^{\prime} X_{(1)}\right)=k_{1 j}^{2}\left(X^{\prime} X\right)+O(1) \text {. }
$$

(2.8) has been proposed previously by Critchley (1985), and, recently, by Wang and Nyquist (1991). They use empirical influence curve considerations. Chaterjee and Hadi (1988) also propose this approximation. Note that corollary 1 allows a direct formal statement for estimating the approximation error directly from the sample size $n$.

\section{Approximations for the eigenvalues of $Z$ ' $Z$}

The main task of this paper is to present the corresponding version of (2.8) for the eigenvalues $\left\{\lambda_{j}\right\}$ of the correlation matrix $Z^{\prime} Z$. Let $\mu \in \mathcal{M}$ such that the functional $\mathrm{C}: \mathcal{M} \longrightarrow \varphi \mathrm{p}$

$$
C[\mu]=\int_{C}[x-m][x-m]^{\prime} \mu(d x)
$$

where $m=\int_{C} x \mu(d x)$, yields a positive definite pxp matrix. Define the functionals $S[\mu]=I_{p}^{*} C[\mu]$, where '*' is the Hadamard product of matrices 
(see Rao (1973), p. 30 for definition), and $\operatorname{CS}[\mu]=S^{-1 / 2}[\mu] C[\mu] S^{-1 / 2}[\mu]$.

It is easy to see that

$$
Z^{\prime} Z=C S\left[F_{n}\right],
$$

and $\lambda_{j}=\ell_{j}\left(\operatorname{CS}\left[F_{n}\right]\right), \gamma_{j}=q_{j}\left(\operatorname{CS}\left[F_{n}\right]\right)$.

Let $h \in \mathcal{M}$. Define the pxp matrices

$$
P(\mu, h)=\int_{C} x x^{\prime} h(d x)-\left[\int_{C} x \mu(d x)\right]\left[\int_{C} x h(d x)\right]^{\prime}-\left[\int_{C} x h(d x)\right]\left[\int_{C} x \mu(d x)\right]^{\prime}
$$

and

$$
\mathrm{R}(\mu, \mathrm{h})=\mathrm{S}^{-1 / 2}[\mu] \mathrm{P}(\mu, \mathrm{h}) \mathrm{S}^{-1 / 2}[\mu]-(1 / 2)\left(\mathrm{S}^{-1 / 2}[\mu] \mathrm{C}[\mu] \mathrm{Q}+\mathrm{QC}[\mu] \mathrm{S}^{-1 / 2}[\mu]\right),
$$

where $Q=I_{p}^{*} P(\mu, h) * S^{-3 / 2}[\mu]$. We proof the following lemma.

Lemma 1. If $C[\mu]$ is p.d. and $\ell_{J}(\operatorname{CS}[\mu])$ is simple, then, $\ell_{J}(\operatorname{CS}[\mu])$ is weakly differentiable at $\mu$ and

$$
\mathrm{D}_{j}(\operatorname{CS}[\mu])(\mathrm{h})=q_{j}^{\prime}(\operatorname{CS}[\mu]) \mathrm{R}(\mu, \mathrm{h}) g_{j}(\operatorname{CS}[\mu]) .
$$

Proof. It is easy to see that $C[\mu+t h]=C[\mu]+t P(\mu, h)+o(|t|)$. On the other hand, a standard first order Taylor expansion yields $\mathrm{S}^{-1 / 2}[\mu+\mathrm{th}]=\mathrm{S}^{-1 / 2}[\mu]+\mathrm{tQ}+\mathrm{o}(|\mathrm{t}|) . \quad$ Therefore,

$$
\operatorname{CS}[\mu+\mathrm{th}]=\operatorname{CS}[\mu]+\mathrm{tR}(\mu, \mathrm{h})+\mathrm{o}(|\mathrm{t}|) .
$$

The rest follows from (3.2) and the perturbation series for $\ell_{j}(\operatorname{CS}[\mu+\operatorname{th}])$.

We now derive the approximation for $\lambda_{J}-\lambda_{J(1)}$.

Theorem 2. Suppose that, for every $n$ and $1 \leq i \leq n$, the matrix $\operatorname{CS}\left[(1-t) F_{n}+\mathrm{tF}_{n-1(1)}\right]$ is p.d. and has all its eigenvalues simple at every point of the segment $0 \leq t \leq 1$. We have, uniformly in $1 \leq j \leq p$ and $1 \leq i \leq n$,

$$
\lambda_{J}-\lambda_{J(1)}=\frac{n}{n-1}\left[k_{1 j}^{2}-\lambda \sum_{j=1}^{p} \gamma_{k j}^{2} z_{1 k}^{2}\right]+o(1 / n) \text {, }
$$


where $\gamma_{j}=\left(\gamma_{1 j}, \ldots, \gamma_{p}\right)^{\prime}$, and $z_{1}^{\prime}=\left(z_{11}, \ldots, z_{1 p}\right)^{\prime}$ is the ith row of the matrix Z.

Proof. Let $\mu=F_{n}, \quad \lambda=F_{n-1(1)}$ and $h=F_{n-1(1)}-F_{n}=(1 / n-1)\left(F_{n}-\delta_{x}\right)$, by (2.4). We need to determine the expression of $D \ell_{j}\left(C S\left[F_{n}\right]\right)(h)$. We have $\mathrm{P}\left(\mathrm{F}_{\mathrm{n}}, \mathrm{h}\right)=\left[X^{\prime} X / \mathrm{n}-\left(\mathrm{x}_{1}-\overline{\mathrm{x}}\right)\left(\left(\mathrm{x}_{1}-\overline{\mathrm{x}}\right)^{\prime}\right] / \mathrm{n}-1\right.$, where $X$ is the $\mathrm{nxp}$ matrix of centered predictors. Therefore,

$$
S^{-1 / 2}\left[F_{n}\right] P\left(F_{n}, h\right) S^{-1 / 2}\left[F_{n}\right]=\left[Z^{\prime} Z-n z_{1} z_{1}^{\prime}\right] / n-1
$$

On the other hand, if $Q=I_{p}^{*} P\left(F_{n}, h\right) * S^{-3 / 2}\left(F_{n}\right)$, we get

$$
S^{-1 / 2}\left[F_{n}\right] C\left[F_{n}\right] Q=\left[Z^{\prime} Z-n Z^{\prime} Z \operatorname{diag}\left(z_{1} z_{1}^{\prime}\right)\right] / n-1 \text {, }
$$

and

$$
Q C\left[F_{n}\right] S^{-1 / 2}\left[F_{n}\right]=\left[Z^{\prime} Z-n d i a g\left(z_{1} z_{1}^{\prime}\right) Z^{\prime} Z\right] / n-1,
$$

where $\operatorname{diag}\left(z_{1} z_{1}^{\prime}\right)$ is the pxp diagonal matrix of $k$ th diagonal element equal to $z_{1 \mathrm{k}}^{2}$. According to lemma $1,(3.3),(3.4)$ and (3.5) lead to

$$
D \ell_{j}\left(C S\left[F_{n}\right]\right)(h)=\left[-n k_{1 j}^{2}+n \lambda_{j} \sum_{k=1}^{p} \gamma_{k j}^{2} z_{1 k}^{2}\right] / n-1 .
$$

We can now parallel the arguments in the proof of theorem 1 to obtain an analog of (2.6) which yields

$$
\max _{1 \leq j \leq m} \max _{1 \leq 1 \leq n}\left|\lambda_{j(1)}-\lambda_{j}-D \ell_{j}\left(C S\left[F_{n}\right]\right)(h)\right| \leq c_{n}\left\|\frac{1}{n-1}\left(F_{n}-\delta_{x_{l}}\right)\right\|_{v^{\prime}}
$$

where $c_{n} \longrightarrow 0$ as $n \longrightarrow \infty$. Since we have $\left\|\frac{1}{n-1}\left(F_{n}-\delta_{x_{1}}\right)\right\|_{v}=2 / n$, the theorem follows.

\section{Applications}

We now relate approximation (3.3) to the detection of collinearity-influential points. 


\subsection{Interpretation}

From (3.3), we see that deletion of the ith case can produce different effects in $\lambda_{j}$. However, several particular cases are worth to observe:

a) If case $i$ is extreme in the jth direction, i.e. $k_{1 j}^{2} / \lambda$, is large (recall decomposition (1.6) for $\mathrm{v}_{11}$ ) we have typically $\mathrm{k}_{1 j}^{2} / \lambda_{j}>\max$ $z_{1 k}^{2}>\sum_{k=1}^{p} \gamma_{k j}^{2} z_{1 k}^{2}$ and, therefore, deletion of case $i$ will tend to produce $\lambda_{j}-\lambda_{J(1)}>0$. If $\lambda_{j}$ is an eigenvalue of large or moderate size, we can deduce that case $i$ might be shading the perception of a collinearity. However, if $\lambda_{J}$ is small, deletion of case $i$ will tend to strengthen the collinearity situation;

b) If case $i$ is approximately orthogonal to $\gamma_{j}$, i.e. $k_{1 j}=z_{1}^{\prime} \gamma_{j} \cong 0$, we will expect that deletion of case $i$ will produce $\lambda_{J}-\lambda_{J(1)}<0$. Therefore, if $\lambda_{j}$ is small, case $i$ might be inducing a collinearity among the columns of $\mathrm{X}$.

\subsection{An example}

We will use for illustration a data set presented by Gunst and Mason (1980, appendix A). The response variable is the GNP (Gross National Product) of 49 countries explained by six socioeconomic variables (see Gunst and Mason (1980) for details and meaning of the variables). These data set have been analyzed in Mason and Gunst (1985) and Belsley (1991, chap. 8).

For diagnostic purposes, approximation (3.3) can be supported with the use of a principal-component plot $\left(\mathrm{k}_{\mathrm{J}}, \mathrm{k}_{1}\right)$. The use of principal-component plots for both detecting extreme cases and 
collinearity is originally proposed by Hocking (1984). See also Velilla (1989).

For these data, we have $\lambda_{1}=0.0267$ which is an indicator of a slight degree of collinearity. Figure 1 shows a principal-component plot $\left(\mathrm{k}_{1}, \mathrm{k}_{5}\right)$. The two starred points are Hong Kong and Singapore which outlie in the $k_{5}$ direction. Since, $k_{5}^{\prime} k_{5}=\lambda_{5}$ these two points are suspicious of inflating artificially the eigenvalue $\lambda_{5}$ and this might be provoking, according to the restriction $\sum_{j=1}^{6} \lambda_{j}=6$, the small value of $\lambda_{1}$ above.

Flgure 1

Table 1 shows the coordinates of $\gamma_{1}$ and the centered and scaled 
coordinates of the cases Hong Kong and Singapore. It can be seen that both Hong Kong and Singapore are, approximately, orthogonal to $r_{1}$. The first summand in the right hand side of (3.3) is dominated by a negative quantity and, therefore, if I=(Hong Kong, Singapore) it can be anticipated that $\lambda_{1}-\lambda_{1(\mathrm{I})}<0$. In fact, $\lambda_{1(\mathrm{I})}=0.1800$ and hence cases Hong Kong and Singapore are creating artificially a collinearity among the six socioeconomic variables.

Table 1

\section{Final coments}

A natural companion of the approximation (3.3) is the extension to multiple cases deletion and the associated masking problems. For (3.3), for example, the aproximation for deletion of the group of cases $I=\left\{i_{1}\right.$, $\left.\ldots, \mathrm{i}_{\mathrm{k}}\right\}$ adopts the form

$$
\lambda_{j}-\lambda_{j(1)}=\frac{n k}{n-k}\left[\sum_{l \in I} k_{1 j}^{2}-\left(\lambda_{j} / k\right) \sum_{1=1}^{p} \gamma_{1 j}^{2}\left(\sum_{1 \in I} z_{11}^{2}\right)\right]+o(1 / n)
$$

Other possible application of the techniques in section 2 refers to the set of condition indexes $\left\{\eta_{\mathrm{J}}\right\}$. It can be shown that, under conditions similar to those appearing in the statement of theorem 2, we have, uniformly in and $1 \leq j \leq p$,

$$
\eta_{J(1)}-\eta_{j}=\frac{n}{2(n-1)} \eta_{j}\left[\left(k_{1 j}^{2} / \lambda_{j}\right)-\left(k_{i p}^{2} / \lambda_{p}\right)+\sum_{k=1}^{p}\left(\gamma_{k p}^{2}-\gamma_{k j}^{2}\right) z_{i k}^{2}\right]+o(1 / n)
$$


Chaterjee and Hadi (1988) have proposed the measure $H_{1}=\left|\kappa_{(1)}-\kappa\right| / \kappa$ as a diagnostic tool for collinearity-influential points. Approximation (5.2), in the particular case of $j=1$, might be used to obtain a natural approximation $\tilde{H}_{1}$ for $H_{1}$. See also Chaterjee and Hadi (1988) for an approach to $H_{1}$ based on a power method for approximating the maximal and minimal eigenvalues of a matrix.

The theory presented in this paper is an extension of the results obtained in Velilla (1988). The functional-based technique presented here can be suitably modified to treat problems in which the design matrix differs from $Z$, whenever the modified cross products matrix can be expressed in terms of a functional depending on the empirical $F_{n}$. For example, Belsley (1991) strongly recommends not centering the data and scaling the columns of $\mathrm{X}$ to unit length to form the matrix $\widetilde{\mathrm{X}}$, say. It is straightforward to express $\widetilde{\mathrm{X}} \widetilde{\widetilde{X}}$ as a functional depending on $F_{n}$ and, as a consequence, analyze, via the techniques exposed in section 2 , the importance of the rows of $\mathrm{X}$ in the collinearity measures related to $\tilde{\mathrm{X}}$.

\section{Ref erences}

BELSLEY, D.A. (1991), Ganditianing Diagnastics, J. Wiley.

BELSLEY, D.A., E. KUH, and E. WELSCH (1988), Regressian Diagnostics: Identifying Influential Data and Yources of Collinearity, J. Wiley. CHATERJEE, S. and HADI, A. S. (1988), Sensitivity Analysis in Linear Regression, J. Wiley.

COOK, R.D. and WEISBERG, S. (1982), Residuals and Influence in Regression, Chapman and Hall.

CRITCHLEY, F. (1985), "Influence in Principal Component Analysis", 
Biametrika 72. 627-636.

GUNST, R.F. (1983), "Regression Analysis with Multicollinear Predictor Variables", Communications in Statistics A12, 2217-2260.

GUNST, R.F and MASON, R.L. (1980), Regression Analysis and its Applications, Marcel Dekker.

KATO, T. (1982), A Yhart Intraduction to Perturbation Theary for Linear Operatars, Springer Verlag.

LANG, S. (1969), Real Analysis, Addison Wesley.

MASON, R.L. and GUNST, R.F. (1985), "Outlier-induced Collinearities", Technametrics 27, 401-407.

RAO, C.R. (1973), Linear Statistical Inference and its Applications, 2nd ed., J. Wiley.

RUDIN, W. (1974), Real and Complex Analysis, McGraw Hill, 2nd Ed.

VELILLA, S. (1988), Contributions to the snalysis of Influence and Multicollinearity in Linear Regression, Ph. D. dissertation, Universidad Complutense, Madrid (Spain).

VELILLA, S. (1989), "A Note on the Behaviour of Augmented Principal-Component Plots in Regression", Communications in Statistics, Theary and Methods 18(1), 331-342.

WALKER, E. (1989), "Detection of Collinearity-Influential Observations", Communications in Statistics, Theary and Methods 18(5), 1675-1690.

WANG, S.G. and H. NYQUIST (1991), "Effects on the Eigenstructure of a Data Matrix When Deleting an Observation", Computational Statistics and Data Analysis 11, 179-188. 
PLOT OF K1 US KE

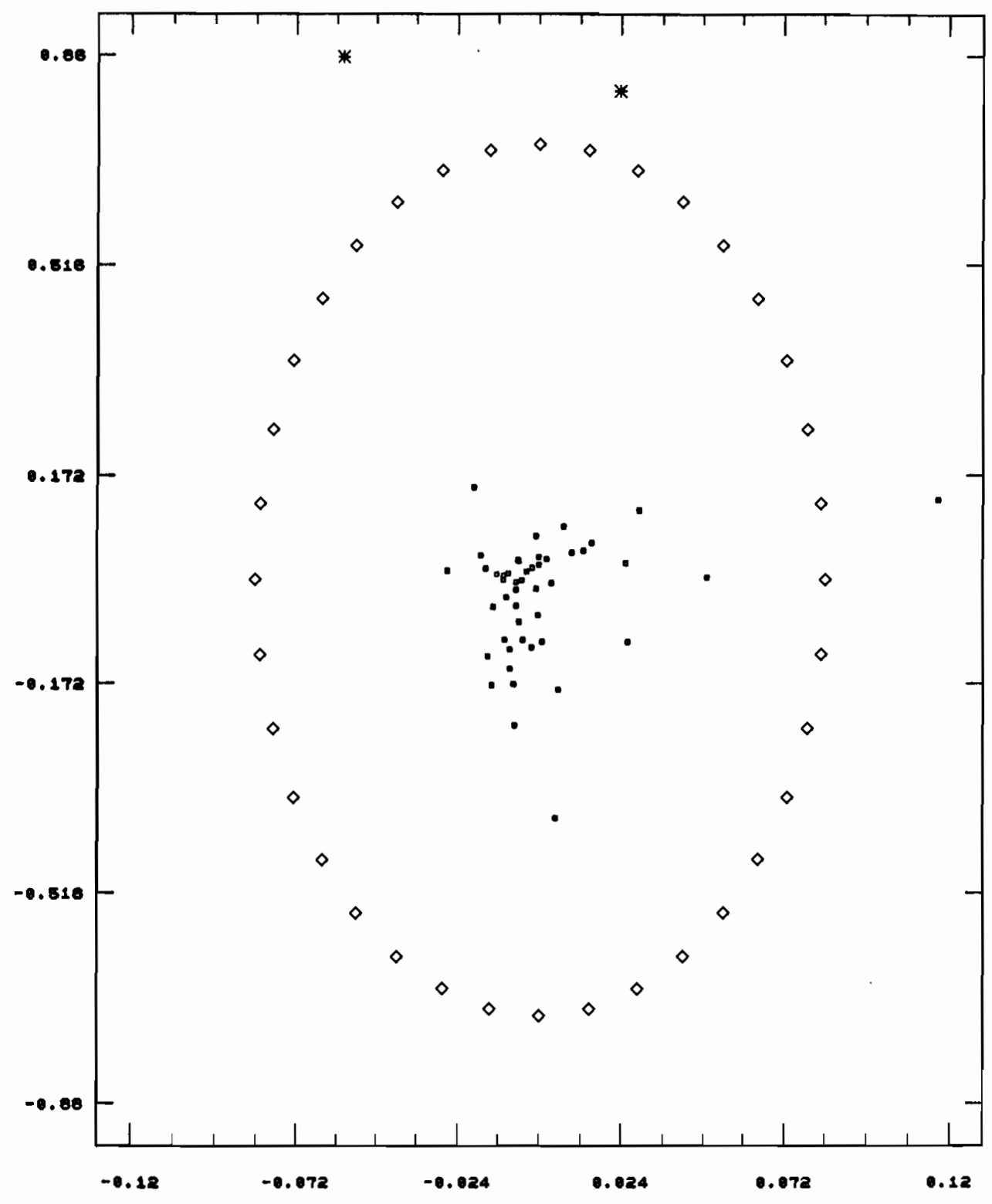

Figure 1 


\begin{tabular}{ccccccc}
\hline Varlable & 1 & 2 & 3 & 4 & 5 & 6 \\
\hline$\gamma_{1}$ & .0066 & .0340 & .7090 & -.7034 & .0275 & .0252 \\
Hong Kong & .0361 & .1301 & .6763 & .6449 & -.1395 & -.0976 \\
Slngapore & -.0730 & .0481 & .6338 & .7158 & -.1869 & .0108 \\
\hline
\end{tabular}

Table 1. Loadings for the eigenvector $\gamma_{1}$ and values of the standardized coordinates of the design matrix for Hong Kong and Sinagapore. 